\section{Combined cataract and trabeculectomy surgery for advanced glaucoma in East Africa; visual and intra-ocular pressure outcomes}

RJC Bowman ${ }^{1,2}$, A Hay $^{3}, \mathrm{ML}$ Wood $^{1}$ and IE Murdoch ${ }^{4}$

\begin{abstract}
Aim To investigate visual and intra-ocular pressure (IOP) outcomes of combined cataract and glaucoma surgery at a high-volume centre in East Africa carried out over a 1-year period (2006).
\end{abstract}

Methods A retrospective analysis of patient records.

Results A total of 163 patients were identified. Mean age was 67 years (SD 11, range 21-86 years) and $113(69 \%)$ were men. Presenting visual acuity in the operated eye was $6 / 60$ or worse in $135 / 163(93 \%)$ and was $<3 / 60$ in 76 of $163(47 \%)$ patients. Mean presenting IOP was $28 \mathrm{~mm} \mathrm{Hg}$ (SD 9, range 12-60). Pre-operative cup disc ratios were 0.8 or worse in 131 of $163(85 \%)$ patients.

Phacotrabeculectomy (PT) was carried out in $130(80 \%)$ cases, small incision cataract surgery trabeculectomy (SICST) in $10(6.1 \%)$ cases, and extra-capsular cataract extraction trabeculectomy (ECCET) in $23(14.1 \%)$ cases. In all, $107(66 \%)$ attended for follow-up (mean interval 104 days, range: 6-390 years, SD 88) and at follow-up $75(70 \%)$ patients had improved visual acuity pre-operatively. Pre-operative cup disc ratio of 0.9 or greater predicted failure to improve VA at follow-up (OR 4.0 95\% confidence interval (CI) 1.30-12.1). Fifty-nine (62\% (95\% CI 52-71\%)) patients had follow-up IOPs of $6-15 \mathrm{~mm} \mathrm{Hg}$ and $82(85 \%$ (95\% CI 78-92\%)) had follow-up IOPs of 6-20 mm Hg.

Conclusion Combined surgery produces visual benefit for most patients with similar pressure control to pure trabeculectomy and is therefore a useful option in practises where follow-up may be doubtful.
Eye (2010) 24, 573-577; doi:10.1038/eye.2009.132; published online 12 June 2009

Keywords: phacotrabeculectomy; Africa; glaucoma; cataract

\section{Introduction}

Earlier audits of our glaucoma practice in East Africa have shown severe disease at presentation, poor acceptance of surgery, poor follow-up post-operatively, and a $20 \%$ risk of visual acuity loss during post-operative followup in those who did return. ${ }^{1,2}$ Other studies from East Africa have reported similar risk of post-operative visual loss ${ }^{3,4}$, and recent work from South Africa suggests that this is usually due to cataract. ${ }^{5}$ One of the reasons for the poor acceptance of trabeculectomy surgery may be that patients are asked to contribute the same fee for a glaucoma operation as for a cataract operation but are also told that, unlike a cataract operation, their vision will not improve as a result of the operation but that they will be protected from going blind.

We hypothesized that combining cataract surgery with glaucoma surgery in those with any significant degree of cataract might allow a small visual benefit even for those with advanced glaucoma, thereby improving compliance with sight-saving glaucoma surgery and conveying the additional benefit of reduced risk of subsequent visual loss because of postoperative cataract development or deterioration (a likely cause of post-operative visual decline). Few data are available on the post-operative outcomes of such combined cataract and glaucoma surgery in Africa. Before a
${ }^{1}$ Comprehensive Community Based Rehabilitation for Tanzania (CCBRT) Disability Hospital, Dar es Salaam, Tanzania

${ }^{2}$ London School of Hygiene and Tropical Medicine, London, UK

${ }^{3}$ Aberdeen University Medical School, Aberdeen, Scotland, UK

${ }^{4}$ Institute of Ophthalmology, London, UK

Correspondence: RJC Bowman,

Comprehensive Community Based Rehabilitation for Tanzania (CCBRT) Disability Hospital,

PO Box 23310,

Dar es Salaam,

Tanzania

Tel: +255222600070

E-mail:

richardandruthbowman@ gmail.com

Received: 14 September 2008

Accepted in revised form: 24 March 2009

Published online: 12 June 2009

Meeting presentation: None 
prospective study in this area, we undertook a retrospective review of cases to determine visual and intra-ocular pressure (IOP) outcomes of combined cataract, and trabeculectomy surgeries in consecutive patients carried out during a 1-year period and to investigate factors affecting these outcomes. We present this retrospective study in this paper.

Comprehensive Community Based Rehabilitation for Tanzania (CCBRT) Disability Hospital is a nongovernmental organization funded unit carrying out around 8000 eye operations per year. It is one of the only two high-volume centres serving a country of 35 million people, meaning that large distances are often travelled by patients for surgery and follow-up.

\section{Materials and methods}

This study is a retrospective 1-year analysis of patients undergoing combined surgery for cataract and glaucoma between January 2006 and January 2007 at CCBRT Disability Hospital Dar es Salaam, Tanzania.

Computerized and paper surgical databases were used to identify the patients. In general, patients who require trabeculectomy (the first-line treatment for primary open-angle glaucoma in our setting), and have any significant cataract (ie, that which might cause visual impairment in an otherwise healthy eye) are considered for combined surgery. Only first eyes of patients who underwent combined surgery in both eyes during this period were included in the study.

Combined phaco-emulsification or trabeculectomy (PT) is the most common procedure carried out for these patients in our unit. RJCB carried out a single-site technique incorporating the fistula into the floor of a superior scleral tunnel (closed with a single releasable suture), and a similar technique for combined small incision (non phaco) cataract surgery and trabeculectomy (SICST) with a wider tunnel, carried out for very dense cataracts with no red reflex. MW carried out two-site technique (temporal clear corneal phaco followed by superior trabeculectomy, closed with two releasable sutures). Extracapsular cataract surgery is combined with trabeculectomy (ECCET) by incorporating a scleral flap into a conventional limbal section. In this study, ECCET was carried out by more junior surgeons who lacked experience in PT or SICST. Releasable sutures are used routinely and 5 fluorouracil (5FU) (either on a soaked cotton swab held subconjunctivally for $5 \mathrm{~min}$ before entering the eye, or injected subconjunctivally at the end of the procedure) is used in all cases. Aurofold hydrophilic acrylic lenses (Aurolab, Madurai, Tamil $\mathrm{Nadu}$, India) are used routinely for PT and a variety of standard PMMA lenses for SICST and ECCET. Twohourly combined dexamethasone and chloramphenicol drops are prescribed routinely for a month postoperatively followed by 4-hourly drops for a month.

Visual outcome was defined as the presence or absence of improvement in visual acuity at discharge and followup, and IOP outcomes were analysed using two success criteria: follow-up IOP ranges of 6-15 and 6-20 $\mathrm{mm} \mathrm{Hg}$. Simple bivariate analysis $\left(\chi^{2}\right.$ and odds ratios with $95 \%$ confidence intervals (CIs) was used to identify risk factors for successful or poor outcomes.

\section{Results}

One hundred and sixty-three eyes in 163 patients were identified. The mean age was 67 years (SD 11, range 21-86 years) and $113(69 \%)$ were men. The presenting visual acuity was 6/60 or worse in 135 of $163(93 \%)$ and was $<3 / 60$ in 76 of $163(47 \%)$ patients. The VA in the operated eye was better than or equal to that of the fellow eye in $111(68 \%)$ cases. Mean presenting IOP was $28 \mathrm{~mm} \mathrm{Hg}$ (SD 9, range 12-60). In 36 cases, presenting IOP was $21 \mathrm{~mm} \mathrm{Hg}$ or less. Eleven (31\%) of these were on some form of anti-hypertensive medication and the remainder were judged to have either primary openangle glaucoma or normal-tension glaucoma according to the fellow eye findings. Cup disc ratios were assessable pre-operatively in 131 cases of which 110 $(85 \%)$ were 0.8 or worse and $77(60 \%) 0.9$ or worse. Most surgery was carried out by two principal surgeons, RJCB $57 \%(n=94)$, MLW 25\% $(n=40)$, and others $18 \%(n=29)$. PT was carried out in $80 \%$ cases $(n=130)$, SICST in $6 \%$ $(n=10)$, and ECCET in $14 \%(n=23$, all of these being carried out by non-principal surgeons). Vitreous loss occurred in five (3\%) cases . Acute post-operative complications were as follows: 14 Shallow AC, 2 Hyphaema, 4 Bleb leaks, 31 Epithelial defects, 11 Other ; 11 of $155(7.1 \%)$ patients had a first-day IOP of above 30. Releasable sutures were removed in 35 cases, the majority in the first 3 days, but up to a period of 2 months post-operatively. At discharge, visual acuity was recorded in 142 eyes, and compared with pre-operation, had improved in 58 (41\%) eyes, was unchanged in 43 $(30 \%)$ eyes, and was worse in $20(29 \%)$ eyes. One hundred and seven $(66 \%)$ patients attended for postoperative follow-up (mean interval 104 days, range: 6-390, SD 88, $n=107), 51(31 \%)$ had 3 months or more follow-up, and 20 (12\%) had 6 months or more follow-up.

Of the 107 who attended for follow-up, corrected visual acuity data were available for 37 and uncorrected for the remainder. Using the best acuity recorded in the files at follow-up (Figure 1a), 75 (70\%) patients had improved their acuity compared with pre-operation by at least one line; $40(37 \%)$ achieved $6 / 18$ or better, and 71 $(66 \%)$ achieved $6 / 60$ or better. Of those with at least 3- and 6-month follow-up, 42 of 51 (82\%) patients and 17 

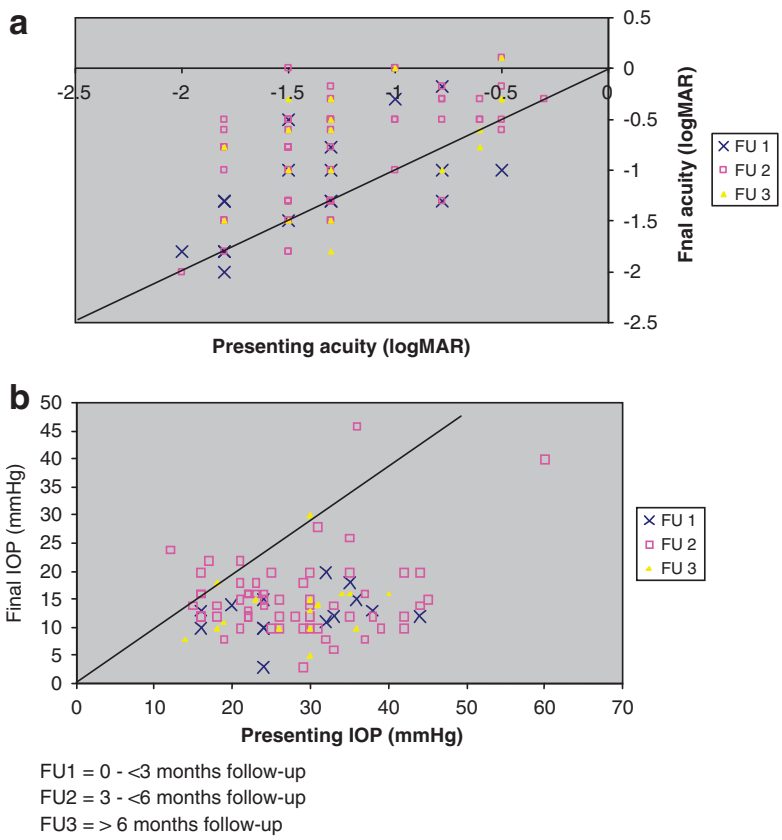

Figure 1 (a) Final follow-up by presenting visual acuity (logMAR). (b) Final follow-up by presenting intra-ocular pressure $(\mathrm{mm} \mathrm{Hg})$.

of $20(85 \%)$ improved their acuity. One patient lost three lines of acuity (high IOP and corneal oedema at followup), and one lost two lines (posterior capsule opacity), the others being either unchanged $(n=21)$ or losing one line $(n=9)$. Having a pre-operative cup disc ratio of 0.9 or greater predicted failure to improve VA at follow-up (OR 4.0 95\% CI 1.30-12.1). Type of operation, surgeon, and presence of intra-operative or post-operative complications had no significant effect on visual outcome at discharge or follow-up. There were no inter-surgeon differences in prevalence of intra- or post-operative complications identified. Pre- and post-operative IOP values are shown in Figure 1b. Ninety-six patients had follow-up IOPs and $59(62 \%$ (95\% CI 52-71\%)) had IOPs of $6-15 \mathrm{~mm} \mathrm{Hg}$ and 82 (85\% (95\% CI 78-92\%)) had IOPs of $6-20 \mathrm{~mm} \mathrm{Hg}$. Of those with at least a 3-month followup, 25 of $48(52 \%(95 \%$ CI 38-66\%)) patients had IOPs of 6-15 mm Hg and 39 of 48 (81\% (95\% CI 70-92\%)) patients had IOPs of 6-20 mm Hg at final follow-up. Of those with at least a 6-month follow-up, 11 of 19 (58\% (95\% CI $26-80 \%))$ patients and 16/19 (84\% (95\% CI 68-100\%)) patients had IOPs of 6-15 and 6-20 $\mathrm{mm} \mathrm{Hg}$, respectively, at final follow-up. Mean follow-up IOP was $15 \mathrm{~mm} \mathrm{Hg}$ (range 3-46, SD =7). There was no significant difference in mean final follow-up IOP between those with and without 3 or 6 months of follow-up. Of the 15 patients who had presenting IOPs of $21 \mathrm{~mm} \mathrm{Hg}$ or less and had final follow-up IOPs, mean pre-operative IOP was $18 \mathrm{~mm} \mathrm{Hg}$ ( $3 \mathrm{~mm} \mathrm{Hg}$ ) and mean post-operative IOP was
$15 \mathrm{~mm} \mathrm{Hg}(5 \mathrm{~mm} \mathrm{Hg}), t=1.8, P=0.10$. ECCET, as opposed to SICST or PT, was associated with failure to achieve follow-up IOP of 6-15 mm Hg (OR $10.195 \% \mathrm{CI}$ 2.6-38.7), and failure to achieve follow-up IOP of 6-20 mm Hg (OR 5.4 95\% CI 1.6-18.8). There was no difference in IOP outcomes between the principal surgeons . Non-attenders for follow-up differed in only one respect from attenders - they were less likely (RR 2.18 95\% CI 1.65-2.87) to live in Dar es Salaam, which was not a predictor of visual or IOP outcome among those who did attend for follow-up. They did not differ in sex, age, or any of the parameters that predicted outcome follow-up.

\section{Discussion}

To our knowledge, this is the first report of the results of a combined glaucoma and cataract surgery in a subSaharan African population with its typically advanced disease. It is a retrospective study with losses to followup typical of our practice (despite various efforts to improve it, including close liaison with community eye workers, regular and repeated dedicated CCBRT bus trips to the same areas, and offers to reimburse travel where necessary), which limits the precision of our findings. Reassuringly, the subgroups of patients with longer follow-up showed sustained pressure control and visual improvement, and there were no significant baseline differences between these subgroups and those with a shorter follow-up. It is this poor follow-up, so common in our setting, and the severely limited accessibility to specialist eye care throughout subSaharan Africa that presents a potential benefit from combined cataract and glaucoma surgery at one sitting over a staged procedure (either cataract or trabeculectomy first) in which the patient may default from the second stage and hence lose vision. An earlier literature review suggested that there is an insufficient evidence to conclude whether staged or combined procedures give better pressure control in glaucoma. ${ }^{6}$ Cataract surgery alone has been reported to produce a small reduction in IOP even in advanced glaucoma, ${ }^{7}$ but the mean drop was only $4 \mathrm{~mm} \mathrm{Hg}$ and most patients were still on topical medication post-operatively, suggesting it to be an inadequate procedure for our population. Our results in terms of IOP control were similar to our earlier report of simple trabeculectomy ${ }^{2}$ (which achieved 73\% (CI 66-80\%) post-operative IOPs of $15 \mathrm{~mm} \mathrm{Hg}$ or less and 90\% (CI 85-95\%) $21 \mathrm{~mm} \mathrm{Hg}$ or less, mean follow-up 8 months). The trabculectomy group had a slightly younger population (mean age 61 years vs 67 years for this study) with better presenting vision (only $19 \%$ eyes blind pre-operatively vs $47 \%$ in this study) but 
similar optic neuropathy severity ( $83 \%$ had cup disc ratios of 0.8 or worse pre-operatively).

We aim for an IOP of 15 or less in these patients because of their advanced disease and achieved this in $66 \%$ of the cases. This is a similar proportion to a series from the $\mathrm{UK}^{8}(62 \% \leqslant 16 \mathrm{~mm} \mathrm{Hg})$; however, the follow-up was much longer in the UK group (mean follow-up 43 months). The secondary target of $20 \mathrm{~mm} \mathrm{Hg}$ was achieved in $90 \%$. ECCET was less likely to achieve this target pressure than PT or SICST, probably because of greater conjunctival dissection and more post-operative inflammation, but as all the ECCETs were carried out by more junior surgeons and all the PTs and SICSTs were carried out by the principal surgeons, we cannot distinguish the effect of surgical experience $v s$ operation type in this study. Likewise, any comparison between single and two-site PT is confounded by the surgeon effect, as all single-site operations were carried out by one principal surgeon and all two-site operations by the other - there were no significant differences in visual or IOP outcomes or complication rates between principal surgeons, and therefore between single- and two-site techniques. An earlier report of PMMA vs silicone IOLs for PT also found higher post-operative IOPs associated with larger wound size, ${ }^{9}$ though this has been questioned in a more recent study in which there was no difference..$^{10}$ Phacoemulsification remains uncommon in Africa; however, small incision non-phaco (scleral tunnel) cataract surgery is gradually overtaking traditional ECCE even among non-physician cataract surgeons, and converting a superior scleral tunnel into a trabeculectomy is easier than for a traditional large limbal incision. Owing to the small number of SICSTs carried out in this study, more information is required about the outcomes of this operation, including from other centres, especially as phacoemulsification is available in relatively few African centres. Further work is also needed to try to improve IOP outcomes in glaucoma ( \pm cataract) surgery in Africa, and a randomized controlled trial of $\beta$ radiation (vs 5 fluorouracil) as an adjunct to combined surgery is now underway in our unit, which includes both PT and SICST. Outcome measures will include IOP and visual acuity, and fieldworkers will be used to improve follow-up. There is increasing interest in non-penetrating alternatives to trabeculectomy, and a recent randomized controlled trial reported better 1-year IOP control after combined phacoemulsification and deep sclerectomy compared with PT. ${ }^{11}$ Although this may show promise for the future in Africa, there are currently very few surgeons familiar with the technique and other authors have reported high rates of secondary procedures to be necessary, such as YAG laser gonioplasty, ${ }^{12}$ which would make the technique less applicable in our setting.
Visual outcomes were encouraging, considering the advanced glaucomatous optic neuropathy, with $70 \%$ of those who returned for follow-up achieving an improvement in visual acuity and nearly $40 \%$ achieving $6 / 18$ or better. An earlier small study of phacotrabeculectomy (PT) in patients with relatively advanced glaucoma from South Africa showed that 6 of 8 patients improved their acuity. ${ }^{13}$ Their optic neuropathy was not as severe as ours and advanced cupping was found to predict failure to improve acuity in our study. Other reports from centres in developed countries with less advanced disease have reported higher proportions of patients improving their acuities (96\% in USA, ${ }^{14} 80 \%$ in Finland ${ }^{15}$ ). Another report of cataract surgery alone in patients with advanced (by western standards)

glaucomatous field loss found generalized improvement in visual field parameters. ${ }^{16}$ Therefore, measuring visual acuity outcomes alone may underestimate the visual benefit to the patient from this procedure. We do not have facilities for visual field measurement at our unit. Even in the absence of improvement in visual acuity, the operation may protect patients against future loss of acuity from both glaucoma (most of our advanced glaucoma cases have poor acuity due to optic neuropathy) and cataract.

The high rate of corneal epithelial defects occurred during a 2-3-month period and was thought to be because of a limited batch of over-concentrated povidone iodine and is now seen much less frequently.

In summary, preliminary reports of trabeculectomy combined with small incision cataract surgery techniques in an East African population with advanced glaucoma are encouraging with acceptable pressure control, visual improvement in a majority of patients, and probable protection from future visual decline. The poor follow-up limits the precision of the findings but also means that a 'one stop' operation for glaucoma and cataract may be a viable and practical approach to management in this setting.

\section{Acknowledgements}

The authors thank CBM for their help in funding this work.

\section{Conflict of interest}

The authors declare no conflict of interest.

\section{References}

1 Mafwiri M, Bowman RJ, Wood M, Kabiru J. Primary openangle glaucoma presentation at a tertiary unit in Africa: intraocular pressure levels and visual status. Ophthalmic Epidemiol 2005; 12(5): 299-302. 
2 Kabiru J, Bowman R, Wood M, Mafwiri M. Audit of trabeculectomy at a tertiary referral hospital in East Africa. J Glaucoma 2005; 14(6): 432-434.

3 Yorston D, Khaw PT. A randomised trial of the effect of intraoperative 5-FU on the outcome of trabeculectomy in east Africa. Br J Ophthalmol 2001; 85(9): 1028-1030.

4 Quigley HA, Buhrmann RR, West SK, Isseme I, Scudder M, Oliva MS. Long term results of glaucoma surgery among participants in an east African population survey. $\mathrm{Br} \mathrm{J}$ Ophthalmol 2000; 84(8): 860-864.

5 Kirwan JF, Cousens S, Venter L, Cook C, Stulting A, Roux P et al. Effect of beta radiation on success of glaucoma drainage surgery in South Africa: randomised controlled trial. BMJ 2006; 333(7575): 942.

6 Jampel HD, Friedman DS, Lubomski LH, Kempen JH, Quigley $\mathrm{H}$, Congdon $\mathrm{N}$ et al. Effect of technique on intraocular pressure after combined cataract and glaucoma surgery: An evidence-based review. Ophthalmology 2002; 109(12): 2215-2224.

7 Altmeyer M, Wirbelauer C, Häberle H, Pham DT. Cataract surgery in patients with end-stage glaucoma. Klin Monatsbl Augenheilkd 2006; 223(4): 297-302.

8 Chang L, Thiagarajan M, Moseley M, Woodruff S, Bentley $\mathrm{C}$, Khaw PT et al. Intraocular pressure outcome in primary $5 \mathrm{FU}$ phacotrabeculectomies compared with 5FU trabeculectomies. J Glaucoma 2006; 15(6): 475-481.

9 Lyle WA, Jin JC. Comparison of 3 and $6 \mathrm{~mm}$ incision for combined phacoemulsification and trabeculectomy.

Am J Ophth 1991; 111(2): 189-196.
10 Junior ES, Wishart PK. Comparison of PMMA, foldable silicone and foldable acrylic hydrophobic intraocular lenses in combined phacoemulsification and trabeculectomy. Arq Bras Oftalmol 2005; 68(1): 29-35.

11 Lüke C, Dietlein TS, Lüke M, Konen W, Krieglstein GK. A prospective trial of phaco-trabeculotomy combined with deep sclerectomy vs phaco-trabeculectomy. Graefes Arch Clin Exp Ophthalmol 2008; 246(8): 1163-1168. Epub 2008 Apr 15.

12 Anand S, Anand N. Combined phacoemulsification and deep sclerectomy (PDS) with intraoperative mitomycin C (MMC) augmentation. Eye 2008; 22(8): 1040-1049. Epub 2007 Apr 20.

13 Gous PN, Roux P. Preliminary report of sutureless phacotrabeculectomy through a modified self-sealing scleral tunnel incision. J Cataract Refract Surg 1995; 21(2): 160-169.

14 Stark WJ, Goyal RK, Awad O, Vito E, Kouzis AC. The safety and efficacy of combined phacoemulsification and trabeculectomy with releasable sutures. Br J Ophthalmol 2006; 90: 146-149.

15 Peräsalo R, Flink T, Lehtosalo J, Ralli R, Sulonen J. Surgical outcome of phaco-emulsification combined with trabeculectomy in 243 eyes. Acta Ophthalmol Scand 1997; 75(5): 581-583.

16 Koucheki B, Nouri-Mahdavi K, Patel G, Gaasterland D, Caprioli J. Visual field changes after cataract extraction: the AGIS experience. Am J Ophthalmol 2004; 138(6): $1022-1028$. 